\title{
Structural dynamic modification using additive damping
}

\author{
B C NAKRA
}

\author{
Mechanical Engineering Department, Indian Institute of Technology, New \\ Delhi, India \\ e-mail: bcnakra@mech.iitd.ernet.in
}

\begin{abstract}
In order to control dynamic response in structures and machines, modifications using additive viscoelastic damping materials are highlighted. The techniques described for analysis include analytical methods for structural elements, FEM and perturbation methods for reanalysis or structural dynamic modification for complex structures. Optimisation techniques used for damping effectiveness include multi-parameter optimisation techniques and a technique using dynamic sensitivity analysis and structural dynamic modification. These have been applied for optimum dynamic design of structures incorporating viscoelastic damping. Some current trends for vibration control are also discussed.
\end{abstract}

Keywords. Structural modifications; viscoelastic damping; perturbation; sensitivity analysis; optimization; vibration control.

\section{Introduction}

Vibrations in machines and structures, if not properly controlled, may cause component fatigue and human discomfort. Some of the methods for vibration control are: reduction of excitation at the source by balancing and alignment in rotating systems and by proper design as in flow systems, avoiding resonances by change in mass and/or stiffness, use of vibration absorbers and incorporation of damping. In the case of aerospace systems and vehicles, the excitations cover a wide frequency range. In such cases, a number of resonances of the structural elements get excited and thus avoiding the resonances may be difficult. The use of damping, however, reduces the vibratory response and dynamic stresses by dissipation of energy and so is being increasingly used as a design parameter rather than being applied after the problem is encountered during operation.

The availability of polymeric materials which are essentially viscoelastic with high damping, has made it possible to effectively control vibratory response. Such materials cannot be used on their own, due to strength and rigidity reasons, and hence a composite construction of metal and polymers can achieve both high damping and strength. These techniques have been used in aerospace and automotive applications and also in machine tools, ships, turbines, electronic and optical devices etc. A number of high damping viscoelastic materials have been developed in the past two to three decades, with a number of applications reported since the conference in 1978 (Rogers 1978) on Damping Technology in the 1980's. 
Analysis of structural elements or structures subjected to dynamic excitation, is usually done by theoretical modelling followed by experimental verification. For complex structures, finite element methods (FEM) are usually employed. The influence of various parameters is quite cumbersome to analyse in view of the large number of re-solutions required to obtain optimum modification. The addition of viscoelastic damping material to a system changes its mass, stiffness and damping. Thus, efforts are made to avoid re-solution to arrive at an optimum design. This is done by structural dynamic modification techniques like the perturbation method. In such cases, changes in modal properties like eigenvalues and eigenvectors due to modifications can be expressed directly in terms of eigen properties of the original unmodified system and changes in mass and stiffness matrices etc. The perturbation method based on FEM has been applied in the present work and is seen to be computationally efficient and accurate when compared with the results obtained by re-solution.

The number of parameters is large and additive damping by use of viscoelastic materials (VEMs) should be used at the optimum location with minimum increase in mass for the desired damping effectiveness. Hence, optimum dynamic design techniques are useful and have been included in the present work. These cover multi-parameter optimisation techniques, dynamic sensitivity analysis (DSA) and algorithms involving use of structural dynamic modification (SDM). The techniques have been applied in order to achieve the desired dynamic characteristic (DDC), viz. system loss factor or vibratory amplitude etc. An algorithm based on DSA and SDM techniques has been discussed with illustration. These techniques are essentially passive ones. Emerging trends include the use of both passive and active control techniques for vibration control.

The objective of the present paper is to discuss some of the above topics. Detailed reviews on the subject with exhaustive references to the work of several other authors are given by Nashif et al (1985), Soovre et al (1984), Sun \& Lu (1995), and Nakra (976, 1981, 1984, 1996).

\section{Viscoelastic damping technology}

Damping of a material or member may be represented by its log decrement during free vibrations, and viscous damping ratio or loss factor or $Q$ factor during forced vibrations. In addition, it may also be represented by the ratio of energy dissipated per cycle to the maximum strain energy. A viscoelastic material like polyvinyl chloride (PVC) has the characteristics of both elastic and viscous elements. The dynamic properties of such materials are represented under harmonic vibration conditions by complex moduli. This is due to the phase difference between stress $\sigma$ and strain $\epsilon$ or $\sigma / \epsilon=E_{1}+i E_{2}$ for direct strain and $G_{i}+i G_{2}$ for the case of shear strain.

Loss factor $\eta$ of the material $=E_{2} / E_{1}$ or $G_{2} / G_{1}$ in direct strain or shear strain conditions respectively.

Viscoelastic material properties can be determined experimentally and are seen to be dependent on frequency and temperature, with the values of moduli, increasing with increase of frequency and decrease of temperature. These factors have to be carefully considered during the dynamic design of structures incorporating VEMs. A number of broad temperature range (BTR) materials have been developed which have high damping and whose dynamic properties do not vary appreciably with temperature (Soovre et al 1984; Nashif et al 1985; Sun \& Lu 1995). 

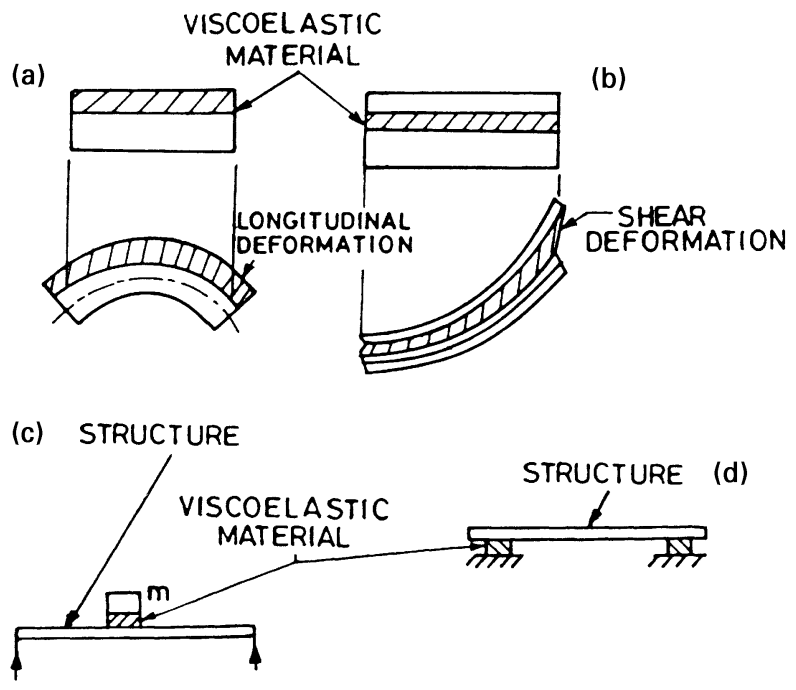

Figure 1. Basic configurations using viscoelastic damping. (a) Unconstrained, (b) constrained treatment, (c) tuned damper, and (d) support damper.

Some of the basic configurations used in practice are shown in figure 1 . The simplest arrangement is the unconstrained one in which a layer of VEM is attached to or bonded to an elastic member. The VEM may be in the form of a spray or sheets. During flexural vibrations of the elastic member, the VEM is subjected to alternate extension and compression. In the case of constrained layer damping arrangement, the viscoelastic layer is constrained between the constraining layer and the base layer and shear strain is induced in the VEM, as shown in figure 1, during flexural vibrations of the base layer. Another technique involves the use of a tuned damper. VEM may also be used at the supports of an elastic member like a shaft. Multilayer arrangements may also be used for vibration damping.

\section{Analysis}

Analysis of structural elements like beams, plates, rings, shells or complex structures consisting of combinations of such elements, involve solution of the governing equations or FEM or structural dynamic methods. These methods have been refined for computational efficiency.

For any mode ' $n$ ', the damping effectiveness of a structure, is denoted by system loss factor $\eta_{s}$, which is obtained from the equation:

$$
\left[-m \omega^{2}+k\left(1+i \eta_{s}\right)\right] q_{n}=f_{n},
$$

where $f_{n}$ is the generalised excitation and $q_{n}$, the corresponding displacement, while $m$ and $k$ are the generalised mass and stiffness respectively. A review of the work on various analysis methods, is available (see Nakra 1976, 1981, 1984, 1996). The system loss factor may be obtained from (1) or by determining the ratio of energy dissipated per cycle to the maximum energy stored during a cycle (Ungar \& Kerwin 1962).

\subsection{Use of governing equations}

Equations for flexural vibrations of the beam are derived for the sandwich or constrained type of arrangement of figure 2. The equations are as below, for all layers assumed as 


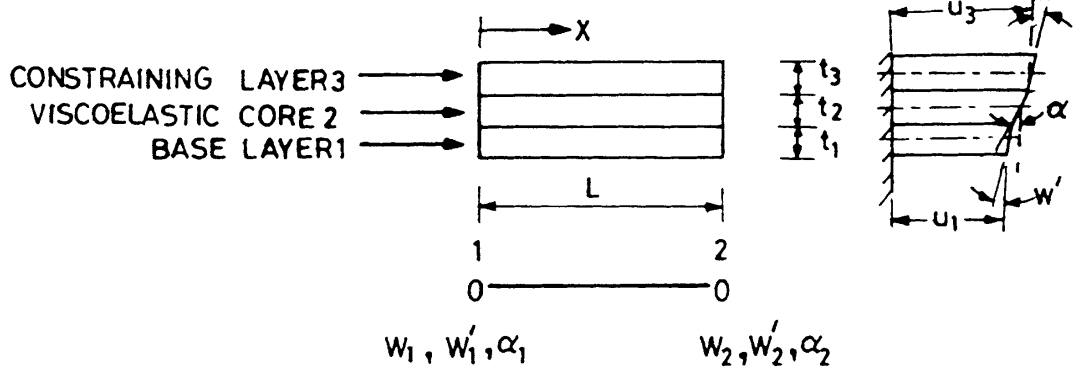

Figure 2. Sandwich beam element with constrained viscoelastic core.

elastic, assuming extension and bending layers 1 and 3 , and shear of layer 2. $E_{1}$ and $E_{3}$ are Young's moduli of layers 1 and 3 respectively, while $G_{2}$ is shear modulus of layer 2 .

$$
\begin{aligned}
& A w^{\prime \prime \prime \prime}-\frac{c a}{t_{2}}\left(w^{\prime \prime} \frac{a}{t_{2}}-u_{3}^{\prime} \frac{m_{1}}{t_{2}}\right)+\rho \ddot{w}=f(x) \sin \omega t, \\
& \frac{c m_{1}}{t_{2}}\left(w^{\prime} \frac{a}{t_{2}}-u_{3} \frac{m_{1}}{t_{2}}\right)+D u_{3}^{\prime \prime}=0 .
\end{aligned}
$$

Also, $u_{3}=-u_{1} m, m=\frac{E_{1} t_{1}}{E_{3} t_{3}}, m_{1}=1+m, b=$ width,

$$
A=\frac{b}{12}\left(E_{3} t_{3}+E_{1} t_{1}^{3}\right), \quad D=b\left(E_{3} t_{3}+E_{1} t_{1} m^{2}\right), \quad c=b t_{2} G_{2} .
$$

For simply supported ends, the following assumed solution satisfies the end conditions,

$$
w=\sum_{n=1}^{\infty} w_{n} \sin \frac{n \pi x}{L} \sin \omega t, \quad u_{3}=\sum_{n=1}^{\infty} u_{3 n} \cos \frac{n \pi x}{L} \sin \omega t .
$$

Writing $f(x)=\sum_{n=1}^{\infty} f_{n} \sin \frac{n \pi x}{L}$, substituting the above in the governing equations, and using complex modulus $G_{2}\left(1+i \eta_{2}\right)$ in place of $G_{2}$, we get an equation similar to (1), from which the expression for $\eta_{s}$ can be obtained as below:

$$
\eta_{s}=H \eta_{2} /\left[\left(1+\alpha \beta^{3}\right)\left(M^{2}+\eta_{2}^{2}\right)+H M\right]
$$

where $H=(3 \psi / \theta)(1+\beta+2 \theta)^{2}\left(1+\eta_{2}^{2}\right)$,

$$
M=(\psi / \theta)[(1 / \alpha \beta)+1]\left(1+\eta_{2}^{2}\right)+1,
$$

shear parameter $\psi=G_{2} /\left[E_{1} t_{1}^{2}(n \pi / L)^{2}\right]$,

$$
\beta=t_{3} / t_{1}, \quad \theta=t_{2} / t_{1} \quad \text { and } \quad \alpha=E_{3} / E_{1} .
$$

A plot of $\eta_{s}$ against $\psi$ as in figure 3 shows that $\eta_{s}$ is maximum only at a certain value of $\psi$. Thus, a change in modal number $n$ or in $G_{2}$ due to temperature or frequency change, may change the damping available. It is seen that for a given increase in size or weight, constrained type arrangement gives higher damping effectiveness than an unconstrained 


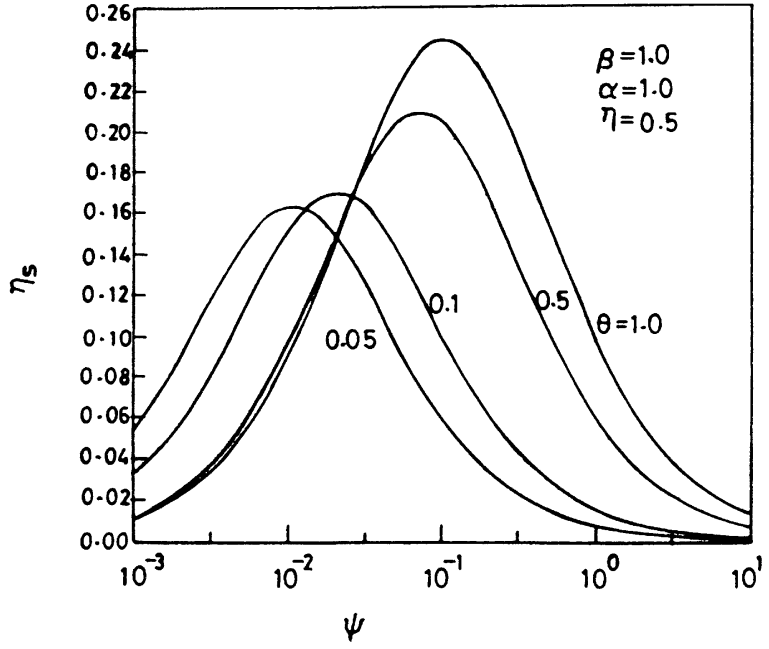

Figure 3. Variation of $\eta_{s}$ with $\psi$ for constrained damping configuration.

one. However, $\eta_{s}$ of the former arrangement depends on modal number $n$ unlike that of the latter arrangement.

In a similar way, the equations of flexural vibrations of a 2-layer unconstrained arrangement of figure 4 may be derived. A plot of $\eta_{s} / \eta$ as in figure 4 shows that system damping increases with increase of $t_{1} / t_{2}$ and $E_{1} / E_{2}$ in general. Asnani \& Nakra (1976) give the analyses and results for multilayer beams with alternate elastic and viscoelastic layers. It is seen that the use of a higher number of layers gives higher $\eta_{s}$ for constant size, weight or static stiffness.
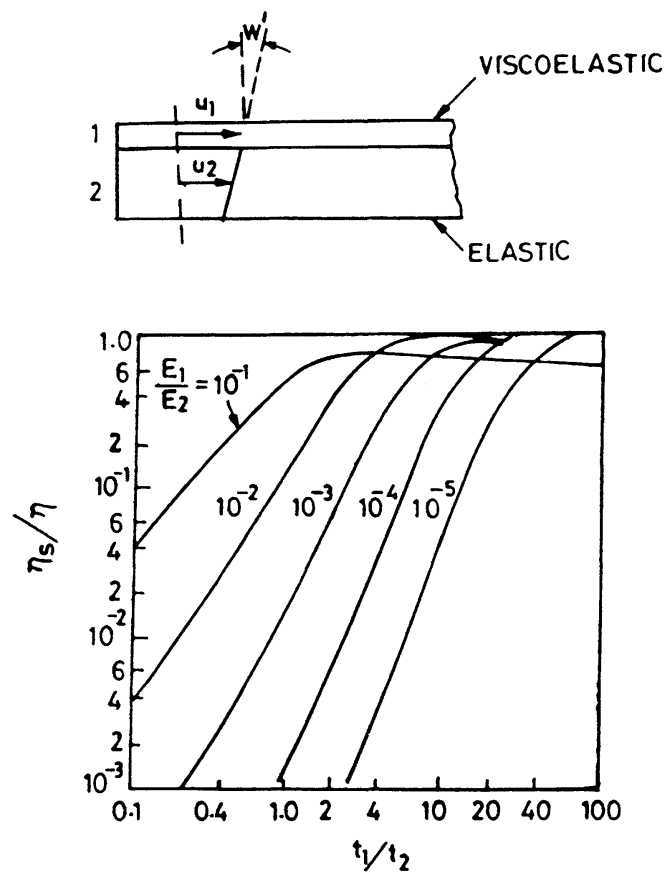

Figure 4. Unconstrained damping configuration. 


\subsection{Finite element method}

Figure 2 shows a sandwich beam element, with nodal displacement $w, w^{\prime}$ and $\alpha . \alpha$ is the rotational displacement in the core and $w^{\prime}$ is flexural angle, with $w$ being the transverse displacement. The stiffness and mass matrices of the element are derived from the expressions of strain energy and kinetic energy respectively, after substituting the expressions for $w$ and $\alpha$ in terms of nodal displacements and shape functions and by the minimisation of the energies (Ravi et al 1993, 1995). The shape functions used are beam-bending shape functions for $w$ and axial bar shape functions for $\alpha$. Complex modulus is used for the shear modulus of the core. A standard eigensolver is used for finding the complex eigenvalues which give natural frequencies and modal loss factors. The response to harmonic excitation is determined by modal summation. The results for the first two resonance frequencies and the maximum amplitude ratio of a fixed-fixed Al-PVC-Al beam obtained by FEM and experiments, are seen to be in good agreement (Ravi et al 1995). The amplitude ratio is the ratio of beam response amplitude to the base excitation amplitude.

For application to a beam with partial coverage as in figure 5, the degrees of freedom for the sandwich element are $w, w^{\prime}$ and $\alpha$ while those for the uncovered elastic element are $w$ and $w^{\prime}$. In order to ensure compatibility, Guyan's reduction was used treating $\alpha$ 's as slaves (Ravi et al 1994).

The method has also been applied to an 'F' structure as in figure 6. Theoretical results were obtained for both in-plane and out-of-plane vibrations, the latter being verified experimentally (Ravi 1994).
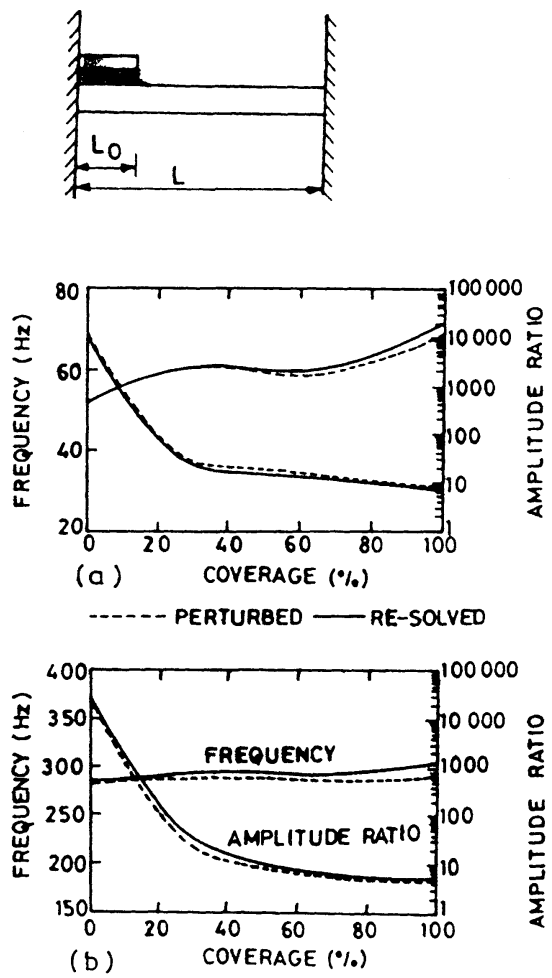

Figure 5. Resonance characteristics of beams with partial converage. 


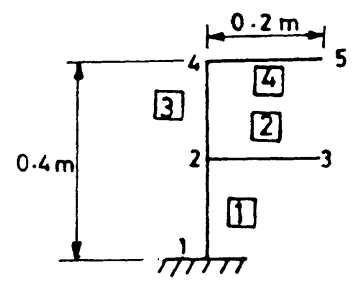

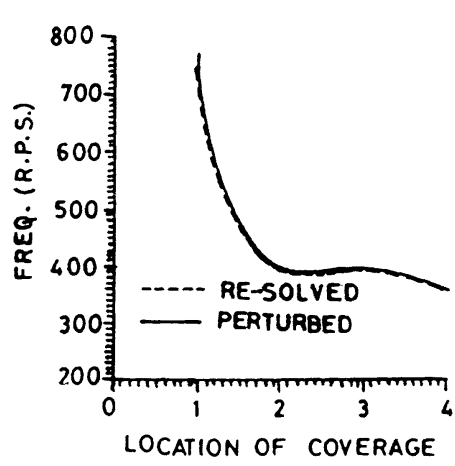

(a)

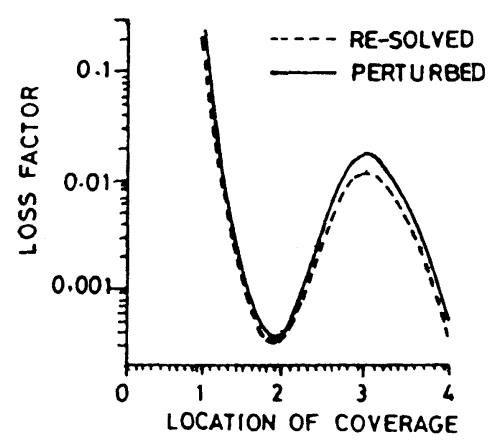

(b)

Figure 6. The $F$-structure and the effect of constrained damping coverage location

\subsection{Structural dynamic modification using perturbation method}

For studying the influence of various damping additions to an untreated structure, perturbation techniques are used, treating modification as a small perturbation to the original structure (Shen \& Stevens 1989). With the above, it is possible to estimate the eigen properties of the modified system by expressing the increments in eigen parameters in terms of increments in stiffness and mass matrices of the system during modification. For obtaining the matrices of the original and modified systems, FEM has been used and for finding the damped natural frequencies and system loss factor, a standard eigenvalue solver is used. Modal summation is then used to find the response to excitation.

The eigenvalue problem may be stated as under,

$$
\lambda_{1}[M]\{\psi\}_{i}=[K]\{\psi\}_{i} .
$$

For a system with modification,

$$
\begin{aligned}
& \lambda_{i}=\lambda_{i}+\Delta \lambda_{i}, \\
& \{\psi\}_{i}=\{\psi\}_{i}+\{\Delta \psi\}_{i},
\end{aligned}
$$

where

$$
\begin{aligned}
\lambda_{i} & =\omega_{1}^{2}\left(1+i \eta_{i}\right) \\
& =\text { eigenvalue for the } i \text { th mode }
\end{aligned}
$$

$\{\psi\}_{i}$ is the corresponding mode shape, $\omega_{i}$ is damped natural frequency and $\eta_{i}$ is modal loss factor of the system. 
The steps for structural dynamic modification or reanalysis are as below.

(1) Solve the eigenvalue problem viz. (5) for the original system.

(2) Find $[\Delta M]$ and $[\Delta K]$ for the modifications. If these are large, divide into smaller ones.

(3) Solve equations for $\Delta \lambda_{i}^{2}$ and $\{\Delta \psi\}_{i}$ for changes $[\Delta M]$ and $[\Delta K]$ using equations given in appendix 1.

(4) Update the modal parameters using (6) and (7).

(5) Repeat steps (2)-(4) till the desired modifications are got.

(6) Solve for $w_{i}$ and $\eta_{i}$ using (8).

Using the above method the results of a partially covered $F$ structure for various constrained type coverages are given in figure 6. The data used are (Ravi et al 1993) $b=t_{1}=0.05 \mathrm{~m}, t_{2}=t_{3}=0.025 \mathrm{~m}$, The base and constraining layers are of mild steel while the core layer has Young's modulus equal to 1/100th that of the base layer, density $1 / 5$ th of the base layer density and material loss factor of 0.6 . The results, using the above SDM method based on perturbation, are seen to be in agreement with those obtained by resolution using FEM. Also, the influence of locations of coverages from 1 to 4 may be seen on the damping effectiveness of the system.

Figure 5 gives similar results for resonant frequencies and amplitude ratio for a partially covered fixed-fixed beam (Ravi et al 1995). The results by both the methods viz. perturbation and resolution are in agreement. The data used are: $L=1.0 \mathrm{~m}, t_{1}=0.01 \mathrm{~m}$, $t_{2}=t_{3}=0.005 \mathrm{~m}, E_{3}=E_{1}=7 \times 10^{10} \mathrm{~N} / \mathrm{m}^{2}, G_{2}=1.962 \times 10^{7} \mathrm{~N} / \mathrm{m}^{2}, \eta_{2}=0.4$. Mass densities $\rho_{1}=\rho_{3}=2700 \mathrm{~kg} / \mathrm{m}^{3}, \rho_{2}=\rho_{1} / 2$. It is seen that in both the cases, perturbation method is very efficient computationally and may be applied to complex structures.

\section{Optimum dynamic design}

\subsection{Multi-parameter optimization}

Multi-parameter optimisation studies are useful since the number of parameters in systems incorporating viscoelastic damping is large.

Optimum design studies for a sandwich plate with partially covered constrained damping treatment, have been carried out (Lall 1984; Lall et al 1987). The Rayleigh-Ritz method has been used for simply supported rectangular plates. Table 1 gives results for additive damping at locations 1,3 and 13 on the plate shown in figure 7 . The results are compared with those of a fully covered plate for the same total mass (Lall 1984). F.C. refers to the fully covered plate and PCL-1 refers to partially covered location 1 . This also applies to PCL-3 and 13. For the base plate, $L=W=0.4 \mathrm{~m}$, thickness $=0.005 \mathrm{~m}$. For the VEM,

Table 1. Comparison of results with optimum values of parameters for fully and partially covered plates.

\begin{tabular}{lcccc}
\hline Values & FC & PCL-1 & PCL-13 & PCL-3 \\
\hline$\omega_{m n}$ & 940.6 & 897.3 & 897.1 & 923.7 \\
$\eta_{m n}$ & 0.048 & 0.024 & 0.043 & 0.054 \\
$P_{L}(\mathrm{~m})$ & 0.4 & 0.24 & 0.19 & 0.16 \\
$P_{w}(\mathrm{~m})$ & 0.4 & 0.3 & 0.35 & 0.38 \\
$t_{3}(\mathrm{~m})$ & $0.5 \times 10^{-3}$ & $0.23 \times 10^{-2}$ & $0.26 \times 10^{-3}$ & $0.28 \times 10^{-2}$ \\
$t_{2}(\mathrm{~m})$ & $0.25 \times 10^{-2}$ & $0.12 \times 10^{-2}$ & $0.72 \times 10^{-3}$ & $0.5 \times 10^{-2}$ \\
\hline
\end{tabular}




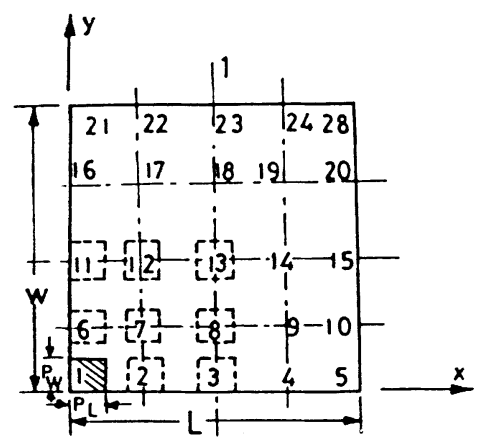

Figure 7. Plate with damped patches.

in-phase shear modulus $=4 \times 10^{6} \mathrm{~N} / \mathrm{m}^{2}$, loss factor $=0.38$ and $m=n=1$. Young's modulus of the base and constraining layer equal $2.07 \times 10^{11} \mathrm{~N} / \mathrm{m}^{2}$ and Poisson's ratio $=0.334 . \eta_{m, n}$ was chosen as the system loss factor for the $m, n$th mode to be maximized, with design parameters $P_{L}, P_{w}, t_{2}$ and $t_{3}$, and the patch coverage area limited to $40 \%$ of the base plate area. The added mass of the patch was equal to that for the fully covered case. It is seen from table 1 that higher system loss factor $\eta_{m, n}$ may be obtained by partial coverage at a suitable location compared to that for a fully covered case.

The algorithm used involved a constrained search strategy (Johnson 1980) in which a constrained problem is made by an unconstrained type by application of external parabolic penalty functions. The $n$-dimensional space is first randomly scanned for a limited number of points and the minimum value is taken as the starting base points. Sequential interval halving is used to find the minimum along the steepest direction. The search is continued till the accuracies on the design variables and the objective function are met.

Another optimisation problem involves maximizing the system loss factor $\eta_{m, n}$ by varying the dimensions of the rectangular patch for the three locations (Lall 1984). Area of the patch equals that of a square patch with $P_{L}=P_{w}=0.15 \mathrm{~m}$. The data is the same as for a previous study with the thickness of the viscoelastic layer $=0.0025 \mathrm{~m}$ and that of the constrained layer $=0.0005 \mathrm{~m}$. Table 2 shows the optimised dimensions of the rectangular patch and the corresponding values of $\eta_{m, n}$ which would be higher than that of a square patch at the same location for PCL-3 and PCL-13. In the above, $m=n=1$.

\subsection{Design sensitivity analysis}

Design sensitivity analysis (DSA) involves evaluation of the sensitivity derivatives of the system dynamic characteristics (DDC) of interest with respect to the chosen design

Table 2. Comparison of results with square and optimum rectangular patches with same covered area.

\begin{tabular}{|c|c|c|c|c|c|c|}
\hline \multirow[b]{2}{*}{ Values } & \multicolumn{2}{|c|}{ PCL-1 } & \multicolumn{2}{|c|}{ PCL-13 } & \multicolumn{2}{|c|}{ PCL-3 } \\
\hline & Square & Opt. rect. & Square & Opt. rect. & Square & Opt. rect. \\
\hline$\omega_{m n}$ & 968.1 & 973.4 & 930.8 & 952.1 & 955.7 & 952.0 \\
\hline$\eta_{m n}$ & $0.184 \times 10^{-2}$ & $0.19 \times 10^{-2}$ & $0.3 \times 10^{-2}$ & $0.77 \times 10^{-2}$ & $0.11 \times 10^{-2}$ & $0.77 \times 10^{-2}$ \\
\hline$P_{L}(m)$ & 0.15 & 0.06 & 0.15 & 0.39 & 0.15 & 0.06 \\
\hline$P_{w}(m)$ & 0.15 & 0.39 & 0.15 & 0.06 & 0.15 & 0.39 \\
\hline
\end{tabular}




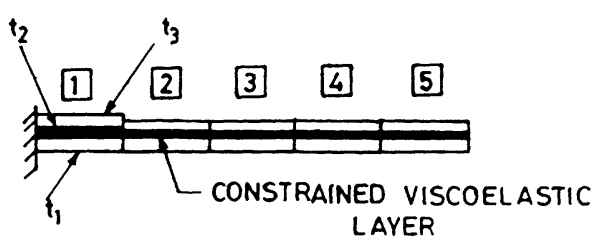

Figure 8. Finite element discretisation of a constrained layer damped beam.

parameters. DSA of structural systems by Haug et al (1986) was based on FEM models. The derivatives of eigenvalues and eigenvectors were derived by Fox \& Kapoor (1968). The analysis is useful in indicating the most effective parameter out of several design parameters for a particular DDC and saves much computational effort. If the value of the sensitivity function is high, the particular parameter effectively changes the DDC.

The sensitivity derivative of the loss factor is derived as below:

Loss factor $\eta, \operatorname{Im}\left(\lambda_{i}\right) / \operatorname{Re}\left(\lambda_{i}\right)$, the numerator and denominator being the real (Re) and imaginary (Im) parts of the complex eigenvalue $\lambda_{i}$. Differentiation with respect to the design parameter $p$ gives:

$$
\frac{\partial \eta_{i}}{\partial p}=\frac{1}{\operatorname{Re}\left(\lambda_{i}\right)}\left[\frac{\partial \operatorname{Im}\left(\lambda_{i}\right)}{\partial p}-\eta_{i} \frac{\partial \operatorname{Re}\left(\lambda_{i}\right)}{\partial p}\right] .
$$

This is normalised by using the logarithmic sensitivity function

$$
S \eta_{i}(p)=\left(\frac{\partial \eta_{i}}{\partial p}\right) /\left(\frac{\eta_{i}}{p}\right)
$$

In a similar way, sensitivity function for other DDC's like natural frequencies, dynamic response etc. may be obtained.

DSA has been applied to a cantilever beam with constrained viscoelastic damping treatment of figure 8. Logarithmic sensitivities of loss factor of the system are plotted in figure 9 for core thicknesses of different elements. Element 1 is seen to be the most sensitive for system loss factor of the cantilever beam with respect to its element core thicknesses.

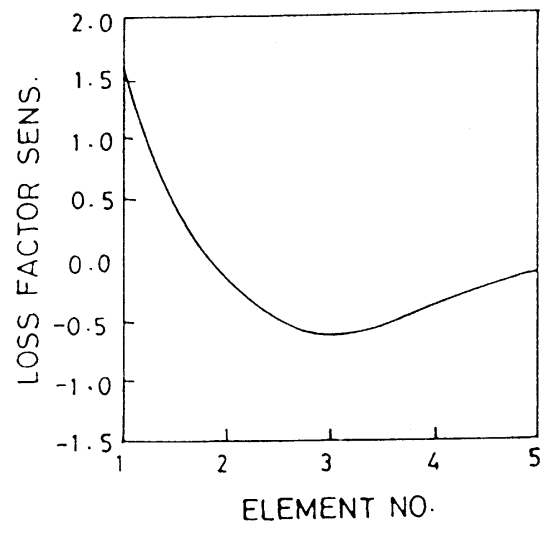

Figure 9. Logarithmic loss factor sensitivities of sandwich cantilever with respect to element core thicknesses. 


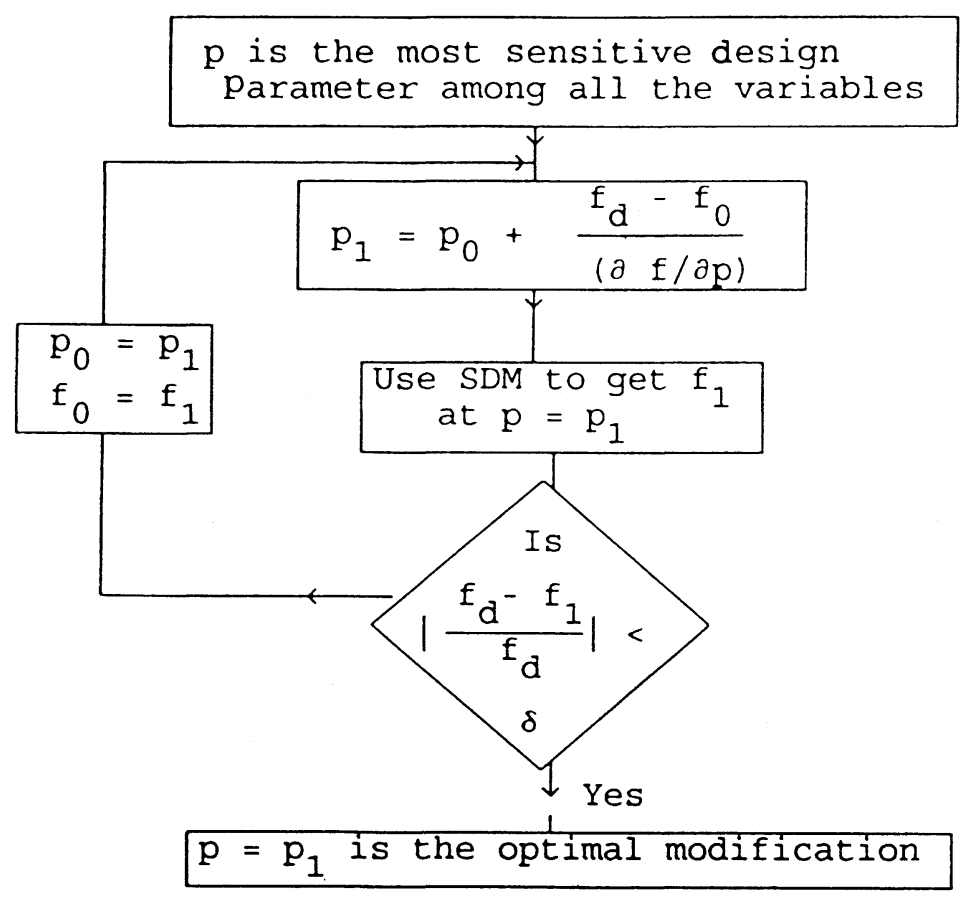

Figure 10. Optimum modification via SDM.

\subsection{Optimum system modification using DSA and SDM}

In the algorithm used, the most sensitive parameter is selected using DSA for the chosen DDC. For a desired change in DDC, the required change in the parameter is determined and the value of DDC is calculated using SDM based on perturbation. The steps involved are given below and the corresponding flowchart is shown in figure 10 .

(1) Calculate the derivatives of the system matrices.

(2) Using the above, compute the logarithmic sensitivities of the DDC with respect to all design variables.

(3) Select the most sensitive parameter for modification.

(4) Calculate the needed changes in the design parameter for required change in DDC.

(5) Use reanalysis or perturbation based SDM to calculate DDC at the modified parameter value.

(6) Test for convergence to the desired DDC value.

(7) If convergence is not obtained, repeat steps (4) to (6) till it is obtained.

(8) If the desired change DDC cannot be obtained as above within acceptable value of design parameter, select the next most sensitive parameter and repeat.

An illustration is given for a cantilever sandwich beam with base layer of aluminium of length $2 \mathrm{~m}$, width and depth $0.05 \mathrm{~m}$ each, Young's modulus $=7 \times 10^{10} \mathrm{~N} / \mathrm{m}^{2}$ and mass density $=2780 \mathrm{~kg} / \mathrm{m}^{3}$. The core is of polyvinyl chloride (PVC) and the constraining layer is of aluminium, both of thickness $0.005 \mathrm{~m}$. The shear modulus of PVC is $2 \times 10^{7} \mathrm{~N} / \mathrm{m}^{2}$, mass density $1390 \mathrm{~kg} / \mathrm{m}^{3}$ and material loss factor 0.5 . The first resonance amplitude ratio 
Table 3. Optimal modification to reduce amplitude ratio using response reanalysis.

\begin{tabular}{lccc}
\hline $\begin{array}{l}P_{k-1} \\
(\mathrm{~m})\end{array}$ & $\begin{array}{c}\text { Resonant frequency } \\
(\mathrm{rps})\end{array}$ & $\begin{array}{c}\text { Resonant } \\
\text { amplitude }\end{array}$ & $\begin{array}{c}P_{k} \\
(\mathrm{~m})\end{array}$ \\
\hline 0.005 & 71.14 & 191.04 & 0.0076 \\
0.0076 & 71.4 & 115.81 & 0.0116 \\
0.0116 & 71.78 & 77.58 & 0.0162 \\
0.0162 & 72.09 & 58.87 & 0.0194 \\
0.019 & 72.19 & 51.66 & 0.0202 \\
0.0202 & 72.2 & 50.17 & - \\
\hline
\end{tabular}

of the beam, viz. the vibration amplitude at the free end to that of the base, is desired to be reduced to 50 from the present value of 191.04. In this case, core thickness of the first element is seen to be the most sensitive parameter for response modification. This is selected for optimum modification as in table 3 and the value of core thickness has to be raised to 0.0202 to achieve the desired value of resonant amplitude (Ravi 1994; Ravi et al 1997).

\section{Current issues}

Some of the current issues are as below.

- Application of optimum dynamic design algorithms to complex systems and over a large frequency range.

- Application of SDM covering a number of modes to updated FEM models.

- Analysis and use of intelligent materials for additive treatment whose damping and stiffness characteristics can change in response to applied voltage, generated by the vibratory response.

- Analysis and use of hybrid treatments of passive and active types.

- Built-in damping at the design stage.

- Refinement of theories to incorporate nonlinear viscoelastic models.

- Development of damping materials whose dynamic materials are least affected by temperature and frequency.

\section{Appendix 1. Perturbation equations for SDM}

The perturbation equations for the changes in eigenvalue, $\Delta \lambda$, and in eigenvector, $\{\Delta \psi\}$, for given changes in stiffness and mass matrices (Shen \& Stevens 1989; Ravi et al 199395; Ravi 1994) are:

$$
\Delta \lambda_{i} \approx \frac{\left\{\psi_{i}\right\}^{T}\left[\Delta K-\lambda_{i} \Delta M\right]\{\psi\}_{i}}{\{\psi\}_{i}[M]\{\psi\}_{i}},
$$

and

$$
\{\Delta \psi\}_{i} \approx \sum_{j=1}^{n} p_{i j}\{\psi\}_{j}, i \neq j
$$


where,

$$
P_{i j}=\frac{\{\psi\}_{j}^{T}\left[\Delta K-\lambda_{i} \Delta M\right]\{\psi\}_{i}}{\left(\lambda_{i}-\lambda_{j}\right)\{\psi\}_{i}^{T}[M]\{\psi\}_{i}}
$$

\section{References}

Asnani N T, Nakra B C 1976 Vibration and damping characteristics of multilayered beams with constrained elastic layers. J. Eng. Ind. 98: 895-901

Fox R L, Kapoor M P 1968 Rates of change of eigenvalues and eigenvectors. AIAA J. 6: 2426-2429

Haug E J, Choi K K, Komkov V 1986 Design sensitivity analysis of structural systems (Orlando, FL: Academic Press)

Johnson R C 1980 Optimum design of mechanical elements (New York: John Wiley \& Sons)

Lall A K 1984 Optimisation studies in viscoelastically damped sandwich beams and plates. Ph D thesis, Indian Institute of Technology, Delhi

Lall A K, Asnani N K, Nakra B C 1987 Vibration and damping analysis of rectangular plate with partially covered constrained viscoelastic layer. J. Vibr. Acoust. Reliab. Des. 109: 241-247

Nakra B C 1976 Vibration control with viscoleastic materials. I. Shock Vibr. Dig. 8: 3-12

Nakra B C 1981 Vibration control with viscoelastic materials. II. Shock Vibr. Dig. 13: 17-20

Nakra B C 1984 Vibration control with viscoelastic materials. III. Shock Vibr. Dig. 16: 17-22

Nakra B C 1996 Vibration control in machines and structures using viscoelastic damping. Emerging trends in vibration and noise engineering (New Delhi: Allied Publishers) pp. 231-246

Nashif A D, Jones D I G, Henderson J P 1985 Vibration damping (New York: John Wiley \& Sons)

Ravi S S A 1994 Structural dynamic modifications and design sensitivity analysis of structures with viscoelastic damping treatment. $\mathrm{Ph} \mathrm{D}$ thesis, Indian Institute of Technology, Delhi

Ravi S S A, Kundra T K, Nakra B C 1993 Eigenvalue reanalysis of damped structures using perturbation method. Proc. of INCARF (New Delhi)

Ravi S S A, Kundra T K, Nakra B C 1994 Eigenvalue reanalysis of sandwich beams with viscoelastic core using perturbation method. Int. J. Anal. Exp. Modal Anal. 9: 203-217

Ravi S S A, Kundra T K, Nakra 1995 A response reanalysis of damped beams using eigenparameter perturbation. J Sound Vibr. 179: 399-412

Ravi S S A, Kundra T K, Nakra B C 1997 Optimal dynamic design using design sensitivity analysis and structural dynamic modifications. Proc. Int. Modal Anal. Conf. XV (Tokyo)

Rogers (ed.) 1978 Proc. Conf. Aerosp. Technol. for the 1980's (Dayton, OH)

Shen S, Stevens K K 1989 A perturbation method for the analysis of structural systems. Proc. of DAMPING-89 (Florida) pp 1-16

Soovre J, Drake M L, Miller V K 1984 A design guide for damping of aerospace structures. Vibration Damping Workshop Proceedings (California) VV1-VV10

Sun C T, Lu Y P 1995 Vibration damping of structural elements (Englewood Cliffs, NJ: Prentice Hall)

Ungar E E, Kerwin E M Jr 1962 Loss factors of viscoelastic systems in terms of energy concepts. $J$. Acoust. Soc. Am. 34: 954-957 